\title{
HIGH-THROUGHPUT SCREENING OF CELL-SURFACE TOPOGRAPHIC INTERACTIONS
}

\author{
Hemant V. Unadkat ${ }^{1 *}$, Marc Hulsman ${ }^{4}$, Kamiel Cornelissen ${ }^{2}$, Bernke Papenburg ${ }^{3}$, \\ Roman K. Truckenmüller ${ }^{1}$, Gerhard F. Post ${ }^{2}$, Marc Uetz ${ }^{2}$, Marcel JT Reinders ${ }^{4}$, \\ Dimitrios Stamatialis ${ }^{3}$, Clemens van Blitterswijk ${ }^{1}$, Jan de Boer ${ }^{1}$ \\ ${ }^{1}$ MIRA Research Institute, Department of Tissue Regeneration, ${ }^{2}$ Discrete Mathematics and Mathematical Programming, \\ ${ }^{3}$ Membrane Science and Technology Group, University of Twente, Postbus 217, 7500 AE Enschede, The Netherlands \\ ${ }^{4}$ Delft Bioinformatics Lab, Delft University of Technology, Mekelweg 4, Delft, the Netherlands
}

\begin{abstract}
High throughput biomaterials screening largely remains unexplored. The present study was an attempt to explore the area of high throughput as well as high content screening of cell surface topographic interaction. An array of surface topographies called TopoChip was fabricated using a widely used polymer in tissue engineering, Poly (DL-lactic acid). The topographies were subsequently screened for their mitogenic effect on cells. We were able to detect topographic parameters responsible for mitogenic effect of surface topographies.
\end{abstract}

KEYWORDS: Biomaterials, High-throughput screening, micro- nanotopographies, cell behavior

\section{INTRODUCTION}

Biomaterials are used in a wide range of clinical applications. In case of implantable biomaterials the response of the human body depends on the cell-material interface resulting in either proper integration or rejection of the implant. Unfortunately nature does not prescribe an optimal surface topography for a given biomedical application ${ }^{1}$. Here we perform high throughput screening of surface topographies using the TopoChip platform to extract, influential topographical parameters. Following iterative in vitro and in silico processes, topographies can be optimized and transferred onto implant surfaces for clinical applications. In the present paper we present topographies that can be used to enhance proliferation of human mesenchymal stem cells.

\section{MATERIALS AND METHODS}

Topographies were designed using randomized algorithms and physically fabricated on a $2 \mathrm{~cm} \mathrm{X} 2 \mathrm{~cm}$ area of a silicon master using photolithography and etching. Hot embossing of Polylactic acid (PLA) sheets was performed using an Obducat Hot embossing/Nano Imprint tool (Obducat AB, Sweden) (Figure 1). 2178 variations in surface topographies each in a area of $290 \mu \mathrm{m}$ X $290 \mu \mathrm{m}$ square area referred to as a TopoUnit were distributed across the TopoChip. Each TopoUnit was repeated twice and finally 4356 TopoUnits constituted a TopoChip.

Chips were placed in a custom designed chip carrier (Figure 3) and EDU-treated 1.2 million/ml human mesenchymal stem cells (hMSC) were seeded onto this chip. The cells were cultured for 8hrs and stained for proliferation (EDU detection) and nuclear stain (TOTO-3) following manufacturer's protocol.

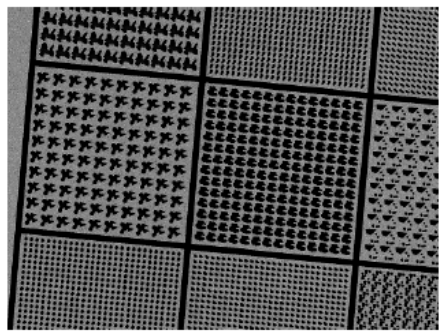

Figure 1. Scanning Electron Micrograph of Patterned TopoChip mold

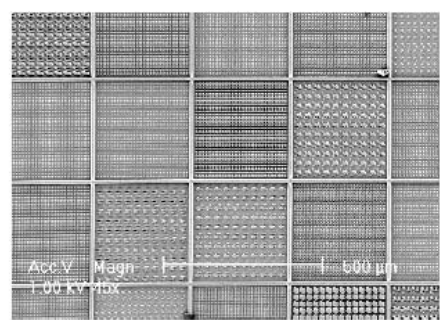

Figure 2. Scanning Electron Micrograph of a part of TopoChip after hot embossing

The chips were imaged using a BD Pathway 435 system with a custom designed macro and the acquired images were subsequently corrected for background, rotation and overlap. The resultant crop images for individual TopoUnits were analyzed using a CellProfiler2 pipeline which involved detection of nuclei (Toto-3 image), detection of proliferating nuclei (EDU/Alexa 488 image), subsequently these two were related and distributed into two bins for proliferating and non proliferating cells. A total of 5 chips were analyzed. 


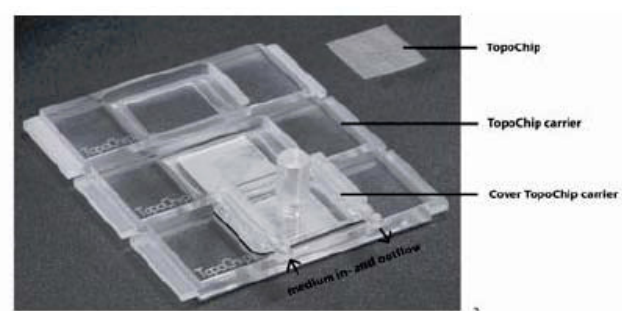

Figure 3. Picture showing TopoChip being loaded onto chip carrier

\section{RESULTS AND DISCUSSION}

We found specific physical topographies that enhance proliferation of the cells. We next used the experimental data to extract influential parameters from each topographical feature. For this, a classifier algorithm was trained on the data, to distinguish between units with high and low measurements (e.g. units with a high or low proliferation ratio). By comparing the $10 \%$ highest versus $10 \%$ lowest measurements, the classifier was tested using a cross validation strategy giving an estimate of the predictive power of the topographical features. Using this in silico approach, we found specific influential parameters (i.e. size of the features) which strongly correlate with experimental results.

We propose that building an evolutionary iterative process between in vitro and in silico experiments shall result in a powerful approach for designing optimal biomaterial interface with wide applications in regenerative medicine or medical devices.
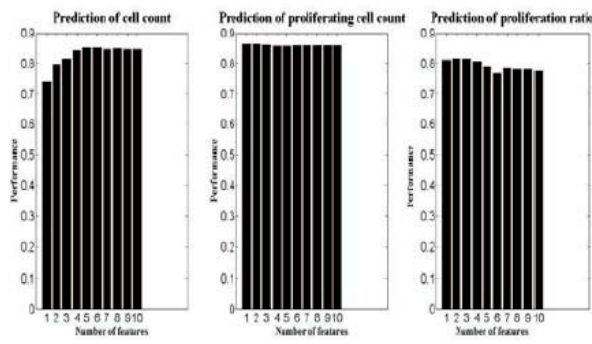

Figure 4. Results from Classifier analysis showing predictability of proliferation behavior of cells with respect to various topographic parameters

\section{CONCLUSION}

We have been able to show that it is possible to perform high throughput as well as high content screening of cell surface topographic interactions.

\section{ACKNOWLEDGEMENTS}

The authors would like to thank Mr. Sip Jan Boorsma and Mr. Dominic Post for help with fabrication of the chip carrier. HVU would like to acknowledge the Marie Curie JOIN(ed)T program for the generous grant. 
Page intentionally left blank 